\title{
Practices and views of neurologists regarding the use of whole-genome sequencing in clinical settings: a web-based survey
}

\author{
Iris Jaitovich Groisman ${ }^{1}$, Thierry Hurlimann ${ }^{1}$, Amir Shoham $^{2}$ and Béatrice Godard ${ }^{\star, 1}$
}

The use of Whole-Genome Sequencing (WGS) in clinical settings has brought up a number of controversial scientific and ethical issues. The application of WGS is of particular relevance in neurology, as many conditions are difficult to diagnose. We conducted a worldwide, web-based survey to explore neurologists' views on the benefits of, and concerns regarding, the clinical use of WGS, as well as the resources necessary to implement it. Almost half of the 204 neurologists in the study treated mostly adult patients $(48 \%)$, while the rest mainly children $(37.3 \%)$, or both $(14.7 \%)$. Epilepsy $(73 \%)$ and headaches $(57.8 \%)$ were the predominant conditions treated. Factor analysis brought out two profiles: neurologists who would offer WGS to their patients, and those who would not, or were not sure in which circumstances it should be offered. Neurologists considering the use of WGS as bringing more benefits than drawbacks currently used targeted genetic testing $(P<0.05)$ or treated mainly children $(P<0.05)$. WGS' benefits were directed towards the patients, while its risks were of a financial and legal nature. Furthermore, there was a correlation between respondents' current use of genetic tests and an anticipation of increased use in the future $(P<0.001)$. However, over half of respondents did not feel sufficiently informed to use WGS in their practice (53.5\%). Our results highlight gaps in education, organization, and funding to support the use of WGS in neurology, and draw attention to the need for resources that could strongly contribute to more straightforward diagnoses and possibly better treatment of neurological conditions.

European Journal of Human Genetics (2017) 25, 801-808; doi:10.1038/ejhg.2017.64; published online 10 May 2017

\section{INTRODUCTION}

The use of Next Generation Sequencing Technologies (NGS), in particular Whole-Genome Sequencing (WGS) and Whole-Exome Sequencing (WES), in genomic and pharmacogenomic research, has sparked considerable debate over the last few years. Ongoing controversies stem from the scientific and ethical issues raised by their use, such as the large amount of data generated, the complexity of its interpretation, the clinical significance of results, the management of incidental results, and the choice of which individual results should ultimately be returned to the participants. ${ }^{1-3}$ More recently, these same discussions have shifted to the use of NGS in clinical settings, giving rise to studies aimed at assessing the attitudes and views of health professionals in various medical fields towards the use of WES and WGS in their practice, and the development of personalized medicine - in particular pharmacogenetics - as well as the return of genetic results, including incidental results, to patients. ${ }^{4-8}$

Neurological conditions form a broad and assorted group of diseases (eg, epilepsy, neuropathy, muscular dystonia, Alzheimer's and Parkinson's diseases, stroke, autism spectrum disorders, intellectual disability, schizophrenia) that are often characterized by highly heterogeneous etiologies. ${ }^{9,10}$ The role genetics can play in understanding these disorders, as well as individual responses to related medications, is increasingly evident. ${ }^{11,12}$ NGS has a particular relevance in neurology diagnostics and therapeutics. Such conditions have been shown to be among the most troublesome to diagnose. ${ }^{13}$ Not only do many of these diseases have a genetic basis, but recent studies highlight the role of de novo mutations as a frequent cause of disorders related to brain development, as well as the contribution of NGS technologies to detect them. ${ }^{13-15}$ The latter thus confirms that neurological disorders are '... amenable to genetic or genomic analysis by next-generation sequencing. ${ }^{14}$ Consequently, an increasing effort is now geared towards developing NGS tools for the diagnosis, prognosis as well as personalized treatment of neurological conditions, and to explicitly promote WES and WGS implementation in the clinical practice of neurology. ${ }^{16-19}$ However, at the very time when such diagnostic tools are being developed, there are scientific and socioethical considerations that may challenge their implementation in neurology clinical settings because of the genetic basis and stigma associated to many neurological condition, which we intended to address in our work. Accompanying the technological advances in detecting the genetic causes of neurological conditions as described above, we deemed it important to examine the opinions and experience of neurologists worldwide about the current and future use of NGS in their practice. As part of a Genome Canada project entitled 'Personalized medicine in the treatment of epilepsy', we developed a web-based survey addressing subjects related to the use of WGS in clinical neurology inquiring on topics such as neurologists' preparedness to request WGS for their patients, or how they

\footnotetext{
${ }^{1}$ Groupe de recherche Omics-Ethics, Institut de recherche en santé publique, Université de Montréal, Montreal, Quebec, Canada; ${ }^{2}$ Département de psychologie, Faculté des arts et des sciences, Université de Montréal, Montreal, Quebec, Canada

${ }^{*}$ Correspondence: Professor B Godard, Department of Social and Preventive Medicine, Université de Montréal, PO Box 6128, Station Centre-ville, Montreal, H3C 3J7, Canada. Tel: +1 5143436111 (\#44036); Fax: +1 514343 2371; E-mail: beatrice.godard@umontreal.ca

Received 15 November 2016; revised 28 February 2017; accepted 28 March 2017; published online 10 May 2017
} 
considered results would impact their patients and families. The results presented herein address neurologists' concerns and their views on the benefits linked to the clinical use of WGS, the training of physicians in the use of NGS, and the resources that would be needed to implement such technologies in their clinical practice.

We developed our study to assess the use of WGS in current practice, identify barriers to its implementation and acknowledge possible current gaps, as WGS is being introduced in the practice of neurology. We believe our findings may help increase insight into the risks and benefits of WGS in clinical neurology, and facilitate its implementation in clinical settings through the development of various resources, such as educational endeavours and guidelines for neurologists.

\section{MATERIALS AND METHODS}

\section{Recruitment}

Neurologists worldwide were contacted through professional associations at international, regional, and national levels. A total of 190 neurology societies and associations in 215 countries/geographical areas were identified and contacted. The list of countries/geographical areas was based on the World Health Organization (WHO) regions (http://www.who.int/countries/fr/). The latter were asked to distribute an invitation to participate in our study. A letter was simultaneously sent to neurologists whose coordinates were publicly available, either on association websites, or as corresponding authors identified through PubMed for the years 2012-2014. Letters were sent to 581 corresponding authors in clinical neurological research worldwide. Neurologists specialized in the treatment of epilepsy registered with the International League Against Epilepsy's website were contacted as well, adding 260 neurologists from the worldwide contact list. We likewise asked participants to forward the invitation to colleagues. Recruitment letters contained a description of the study, privacy and confidentiality measures, and a link to access the online survey. Consent to participate was granted by completing the survey. While letters of invitation were in English, French and Spanish, the survey itself was solely in English. Participants were free to withdraw at any time and were allowed to skip any question. $^{20}$

\section{Sample}

A total of 204 neurologists participated in the study. Almost half treated mostly adult patients $(48 \%)$, the rest mainly children $(37.3 \%)$ or both $(14.7 \%)$. Epilepsy $(73 \%)$ and headaches $(57.8 \%)$ were the main conditions. Most followed patients presenting clusters of neurological conditions. Respondents were based in Europe, North, Central and South America, the Caribbean, South-East Asia, the Western Pacific Region, the Eastern Mediterranean and Africa. Table 1 presents their characteristics.

\section{Survey - instrument}

To lay the groundwork for questions, we reviewed relevant literature and incorporated scientific and/or ethical issues linked to the current state of knowledge, research and practice regarding WGS and neurology. ${ }^{12,14,16-18,21-23}$ One questionnaire targeted neurologists specializing in - or treating - patients with epilepsy, another, those not dealing with epilepsy. Both contained questions addressing respondents' views and perspectives on: (1) clinical practices with genetic/genomic testing (including WGS); (2) circumstances and/or conditions in which WGS should be offered to patients; (3) the potential benefits of the use of WGS; (4) concerns about the use of WGS, (5) the return of results; and (6) needs for training or resources in genomics/genetics. Herein are the comprehensive results, with the exception of return of results, which is the subject of a separate publication.

\section{Data collection}

The first invitation was sent at the beginning of September 2014, and the survey was available until the end of April 2015. Because of snowball recruitment procedures and dissemination by professional organizations, it is impossible to know the total number of neurologists who were invited to participate.
The study was approved by the Research Ethics Board of the Centre hospitalier de l'Université de Montréal (CHUM). Online questionnaires and data collection were conducted by the IT department at Université de Montréal's Public Health Research Institute (IRSPUM). Data collection was anonymous from the researchers' standpoint.

\section{Data analysis}

Information from the completed questionnaires was transferred to an Excel database. Coding and analysis were conducted using the Statistical Package for Social Sciences (SPSS) software, v. 23.0. Descriptive statistics was used to describe sample characteristics. The interpretative analysis, based on $\chi^{2}$ and factor analyses, allowed us to better characterize the respondents and to reach a deeper understanding of what motivated and guided their responses. Two profiles emerged: (a) neurologists who would offer WGS to their patients and (b) those who would not offer WGS to their patients, or did not know about the uses of WGS. A one-way ANOVA was conducted to compare the two profiles and factor analyses with varimax rotation to assess the underlying benefits and concerns on the use of WGS for both profiles. We considered a $P$-value of 0.05 or less as statistically significant.

\section{RESULTS}

Current use of genetic/genomic tools in clinical practice

Neurologists were asked if they used genetic/genomic tests in their practice, namely targeted sequencing, karyotype, WES, WGS and pharmacogenetic tests, and if they foresaw an increase in the clinical use of these tests. Targeted sequencing (44\%) and karyotype (38\%) were the tests used most frequently, while pharmacogenetic tests $(10 \%)$, WES (12\%) and WGS (5\%) were the least used (Supplementary Table 2). We found a correlation between current use of any of these tests and the anticipation of an increased use in the future $(P<0.001)$, with the exception of pharmacogenetic tests. In the short term, respondents anticipated an increase in the use of targeted sequencing and WES. As for the long term, respondents foresaw that the use of WGS was to increase while that of karyotype would not change (Supplementary Table 2). Depending on the test, $12-25 \%$ of respondents answered that they did not know what to expect.

Almost half of 200 respondents were asked by their patients to order a genetic test $(48.3 \%)$. These neurologists indicated that the requests were mainly due to patients' needs to get answers $(58.9 \%)$, or to be sure about the diagnosis and/or prognosis (67.4\%), or still to know whether blood relatives or their children could be at risk (58.9\%). A minority of patients asked for such tests because they had heard about the possibility of ordering such tests through the Internet $(24.2 \%)$. Finally, $28 \%$ of 200 respondents were asked to interpret a test ordered elsewhere.

\section{Circumstances in which WGS should be offered}

We asked participants in what circumstances WGS should be offered to a patient. A very small minority (3.8\%) expressed that WGS should be offered to all patients (Table 1). Over half of the respondents $(61.1 \%)$ stated that it should only be offered to a limited number of patients. The most appropriate indications for offering WGS in clinical practice were to patients with unclear phenotypes (84.9\%), or to those with conditions that seemed to follow a complex inheritance $(84 \%)$, or, in a smaller proportion, to patients at risk of developing comorbidities that may have a genetic background $(76 \%)$, or at risk of pharmaco-resistance (73.4\%; Figure 1). For those responding that WGS should be offered 'in limited circumstances only', we asked in what specific circumstances exactly should WGS be offered to patients with the condition(s) most often seen in their practice. Among various options presented to participants, the reason that was chosen by the 
Table 1 Sample Characteristics

\begin{tabular}{|c|c|c|}
\hline & $\mathrm{N}$ & $\%$ \\
\hline \multicolumn{3}{|l|}{ Gender $\left(\mathrm{n}=155^{\mathrm{a}}\right)$} \\
\hline Female & 80 & 51.6 \\
\hline Male & 75 & 48.4 \\
\hline \multicolumn{3}{|l|}{ Age group $(\mathrm{n}=159)$} \\
\hline 40 years and under & 42 & 26.4 \\
\hline $41-50$ & 54 & 34.0 \\
\hline 51 and over & 63 & 39.6 \\
\hline \multicolumn{3}{|c|}{ Years of clinical practice in neurology $(n=157)$} \\
\hline 10 years and under & 52 & 33.1 \\
\hline $11-20$ years & 51 & 32.5 \\
\hline More than 20 years & 54 & 34.4 \\
\hline \multicolumn{3}{|c|}{ Years of experience in clinical research $(n=156)$} \\
\hline No experience & 27 & 17.3 \\
\hline 10 years and under & 77 & 49.4 \\
\hline $11-20$ years & 32 & 20.5 \\
\hline More than 20 years & 20 & 12.8 \\
\hline \multicolumn{3}{|l|}{ Position in academia $(n=148)$} \\
\hline Full Prof. or equivalent & 18 & 12.2 \\
\hline Associate Prof. or equivalent & 25 & 16.9 \\
\hline Assistant Prof. or equivalent & 38 & 25.7 \\
\hline Fellow & 29 & 19.6 \\
\hline Resident & 6 & 4.1 \\
\hline None & 32 & 21.6 \\
\hline
\end{tabular}

Education/training in genetics/genomics $(\mathrm{n}=159)^{\mathrm{b}}$

Formal

Undergraduate

Graduate

University/Professional lectures

Non Formal

Undergraduate or graduate

Self-study

Practical experience with patients

Characteristics of patients $(n=204)$

Mainly Adults $(n=98)$

Mainly Children $(n=76)$

Both $(n=30)$

Neurological conditions most often treated $(n=204)^{c}$

Cancer

Dementia

Epilepsy

Headaches

Movement Disorders

Multiple Sclerosis

Neurodevelopmental

Neuromuscular

Stroke/vascular neurology

Sufficiently informed to use WGS in clinical practice $(\mathrm{n}=159)$

Yes

Somehow

No

I don't know/l am not sure

Would more resources help you use WGS in your clinical practice? $(\mathrm{n}=159)$

Yes

No

I don't know/l am not sure
Table 1 (Continued)

\begin{tabular}{|c|c|c|}
\hline & $\mathrm{N}$ & $\%$ \\
\hline \multicolumn{3}{|l|}{ Resources that would help you use WGS in clinical practice $(n=129)$} \\
\hline Ongoing training in genomics/genetics & 95 & 73.6 \\
\hline $\begin{array}{l}\text { Increased access to multidisciplinary team (genetic counsellors, } \\
\text { psychologists, and so on) }\end{array}$ & 105 & 81.4 \\
\hline Access to comprehensive and systematically updated databases & 95 & 73.6 \\
\hline Explicit guidelines from professional organizations & 98 & 76 \\
\hline Increased access to sequencing facilities & 65 & 50.4 \\
\hline Other & 3 & 2.3 \\
\hline No answer & 1 & 0 \\
\hline \multicolumn{3}{|l|}{ WGS should be offered... ( $\mathrm{n}=185)$} \\
\hline Only to a limited amount of patients & 113 & 61.1 \\
\hline To ALL patients & 7 & 3.8 \\
\hline NOT be offered & 29 & 15.7 \\
\hline I don't know & 36 & 19.5 \\
\hline No answer & 19 & 9.3 \\
\hline \multicolumn{3}{|c|}{ There are more risks than benefits resulting from the use of WGS in my practice right } \\
\hline \multicolumn{3}{|l|}{ now } \\
\hline Strongly disagree & 36 & 22.8 \\
\hline Somewhat disagree & 43 & 27.2 \\
\hline Generally agree & 30 & 19.0 \\
\hline Strongly agree & 29 & 18.4 \\
\hline I don't know/l am not sure & 20 & 12.7 \\
\hline
\end{tabular}

least number of respondents was a recommendation from a professional organization (56.5\% of 184 respondents; Figure 2).

\section{Risks and Benefits of WGS}

Did neurologists anticipate more risks than benefits resulting from the use of WGS in their practice? From a total of 158 neurologists addressing this question, 50\% disagreed (partially or totally) with the opinion that WGS carried more risks than benefits, while $37.3 \%$ agreed with this view (partially or totally); a small percentage did not know or was not sure about WGS' risk/benefit balance $(12.7 \%)$. Neurologists finding that the use of WGS may bring more benefits than risks treated mainly children $(P<0.05)$. Those who currently used targeted genetic testing $(P<0.05)$, as well as those who had less than 10 years of medical practice $(P<0.05)$ also tended to see more benefit than risk. By contrast, those with more than 20 years of experience tended to see more risks than benefits $(P<0.05)$.

A majority of our participants agreed that, among the benefits that could result from WGS, the following were the most important: detection of new/rare mutations in patients with an uncommon or unclear phenotype (79.4\%), informing patients and their family members about recurrence risks in offspring (78\%), detection of pharmaco-resistance, and determination of appropriate treatment (75.1\%). Among neurologists treating epilepsy on a regular basis, 129 considered that WGS would contribute to the early identification of good candidates for surgery. Among that group, $31.8 \%$ saw it as likely being a benefit for some cases and $15.5 \%$ a benefit in most cases. As for risks, a majority of participants agreed that the cost of WGS, the lack of specific insurance coverage and of insufficient reimbursement (67.3\%), or the lack of access to, or availability of, the test $(62.7 \%)$, and the lack of specific clinical guidelines to use WGS in practice 


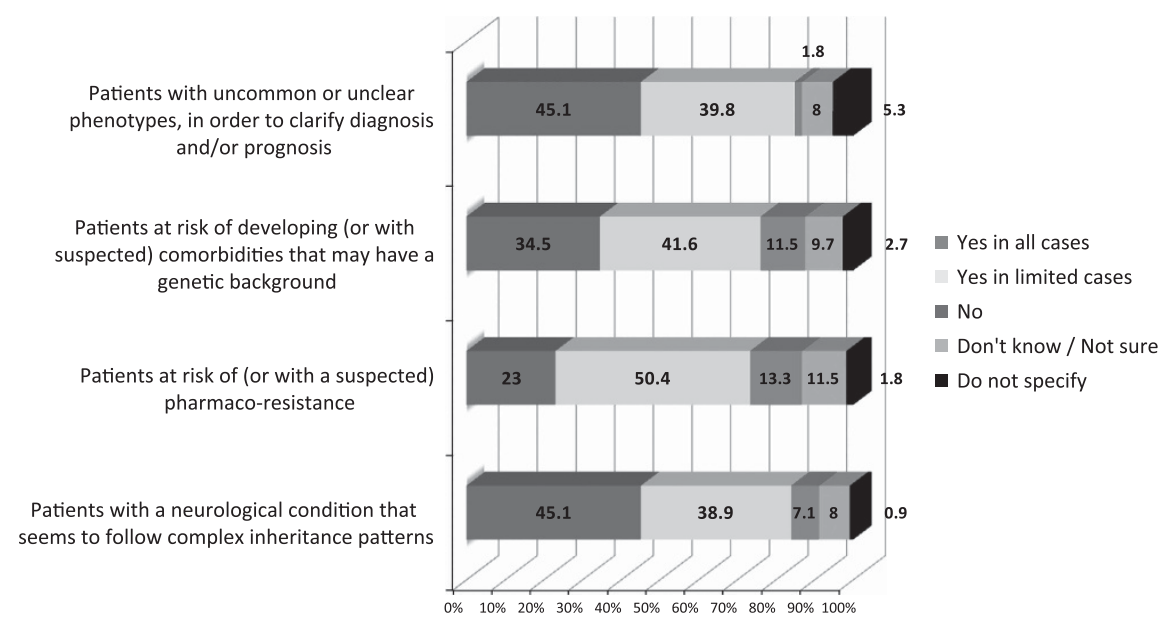

Figure 1 Most appropriate indications for offering WGS in clinical practice.

...after considering what could be the
social and psychological impact of genetic
results on each patient ( $n=181$ )
...if the test results are likely to lead to a
change in the treatment choice and/or
prognosis ( $n=184$ )
...when all other methods have failed to
confirm diagnosis and/or etiology
( $n=186)$
...when recommended by professional
organizations ( $n=184)$
organizations $(n=184)$

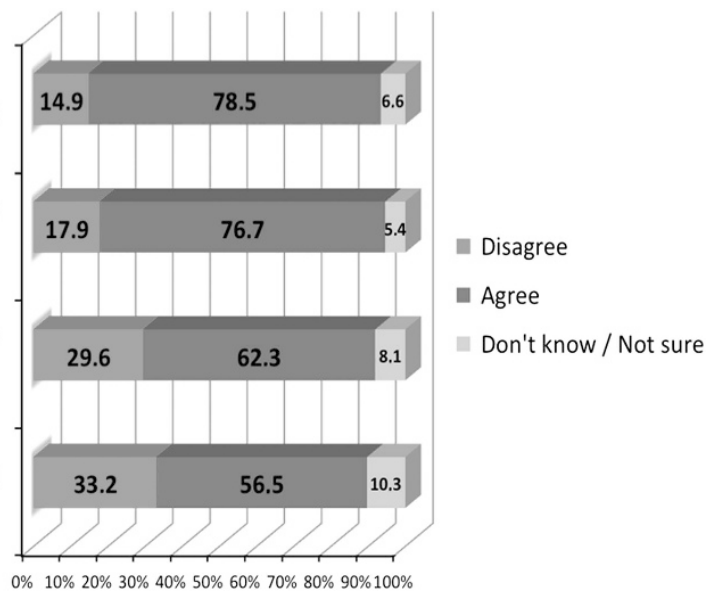

Figure 2 WGS should only be offered in clinical practice.

(47.2\%) were the more concerning risk factors associated with the use of WGS in their practice.

Resources to use genetic tests in neurological practice

It should be noted that more than half of respondents did not feel sufficiently informed to use WGS in the clinic (53.5\%; Table 1). A majority of respondents believed that more resources would help them use WGS in their practice (81.1\%), such as an increased access to multidisciplinary teams (including genetic counselling), or explicit guidelines from professional organizations, access to comprehensive and systematically updated databases or sequencing facilities, or still ongoing training in genomics/genetics (Table 1).

Two Profiles of respondents regarding the use of WGS in clinical practice

On the basis of the circumstances in which respondents considered offering WGS to their patients, our factor analysis brought out two neurologist profiles. 'Profile A' $(58.5 \%)$ would offer WGS to their patients. Profile A includes those that would offer WGS to all their patients and those that would do so in limited circumstances. 'Profile B' $(31.8 \%)$ would not offer WGS or were not sure in which circumstances WGS should be offered. Differences between the two profiles were significant regarding the diagnostic test used in their practice: use of targeted gene sequence $\mathrm{F}(3,169)=2.66, P<0.05$, use of Karyotype F $(3,169)=4.51, P<0.05$, Whole-Exome Sequencing F $(3,147), P<0.05$, and Whole-Genome Sequencing $F(3,174)$, $P<0.05$.

\section{Characteristics of A and B Profiles}

Supplementary Table 3 shows the characteristics of each Profile with respect to the age of patients, neurological conditions most often treated, years of clinical practice in neurology, training in genetics/genomics, and their perception of the degree to which they felt informed on the use of WGS in their clinical practice. Many Profile A physicians followed mainly children, most of them treated epilepsy, and had 20 years of medical practice or less. A total of 97/120 Profile A and 53/65 Profile B respondents indentified their country of residence. Among the Profile A countries, 17/34 are considered by the World Bank as low- to middle-income countries compared to 7/19 Profile B countries (http://data.worldbank.org/ about/country-and-lending-groups).

\section{Benefits of the use of WGS in the clinic by Profile}

Our analysis brought out two factors favouring the use of WGS in clinical practice for both profiles (Table 2): Factor 1 refers to the improvements for the patients and their proxies; Factor 2 refers to the efficiency and effectiveness of WGS. While Factor 1 shows that both A and $\mathrm{B}$ neurologists perceived the same improvements for the patients 
and their proxies, Factor 2 indicates that the only benefit related to the efficiency and effectiveness of WGS shared by both Profiles was to reduce the need for other medical investigations/procedures (Table 2). Profile A neurologists considered the clinical use of WGS helpful to detect new or rare mutations in patients who presented an uncommon or unclear phenotype. Profile B ones saw it as useful to identify genetic risk for comorbidities, and perceived no benefit in storing data (Table 2).

\section{Concerns about the use of WGS in the clinic according to Profile}

The 'Concerns about the use of WGS' produced three factors for Profile A and two factors for Profile B (Table 3). Regarding Factor 1, Profile A neurologists had concerns mainly for patient's proxies, while their Profile B colleagues also had concerns for patients and health professionals, such as issues raised by the management of unexpected findings and risks of discrimination or stigmatization of patients or their family members. Profile B concerns about their patients are also reflected in Factor 2, on the lack of resources to use WGS in the clinic, such as access to genetic counselling, which could lead to emotional harm for patients or proxies. Finally, Profile A neurologists were the only ones to anticipate potential adverse effects of WGS for patients and medical professionals (Factor 3). Even if they would offer WGS to their patients, they were aware of their professional liability for adverse outcomes occuring as a result of returning (or not) the test findings, of the risk of discrimination or stigmatization of patients or their family members, as well as of the risk of inducing emotional harm in patients or proxies.

\section{Need of resources to use WGS in the clinic per profile}

To determine which resources neurologists would fully benefit from when implementing NGS technologies in their practice, we presented a list of possible ones from which to choose. Profile A doctors, who would offer WGS to their patients, expressed a significantly higher need for resources than their Profile B counterparts (Figure 3). When presented with a list of possible resources, Profile A respondents expressed a need for all of them in a higher proportion than those of Profile B (Figure 3), whether in terms of explicit guidelines from professional organizations or increased access to multidisciplinary teams - including genetic counsellors and psychologists - or, still, to databases and sequencing facilities. They also asked for ongoing training in genomics in much greater numbers. And yet, they had more training in genetics and/or genomics - both formal and informal - than Profile B neurologists (Supplementary Table 3). They also considered themselves sufficiently informed to use WGS in their practice in a higher proportion than their counterparts (Supplementary Table 3).

\section{DISCUSSION}

\section{Current and future use of NGS}

The widening use of NGS technologies is expected to help find elusive, causative genetic defects and help in the diagnosis and treatment of a broader array of medical conditions. ${ }^{9,24,25}$ Our results show that WES and WGS, together with pharmacogenetic/pharmacogenomic tests, were still rarely used with our respondents' patients. However, half of respondents felt benefits outweighed risks, which shows that the potential value of NGS in medical practice was well recognized. This was the case for neurologists treating mostly children, probably because the advantages had already been demonstrated in this population. ${ }^{26}$ This is also the case of the 'young' neurologists, trained during a period characterized by major changes in sequencing tools, and by the identification of numerous genes linked to various medical
Table 2 Benefits about the use of WGS in clinical practice by Profile

Profile $A^{a}$
Factor 1: Patient and proxies improvements $B^{b}$
1. Improve the patients' or proxies' psychological well-being
2. Improve patients'/proxies' access to, and help to get better attention in
healthcare and social services
3. Inform patients and family members about recurrence risks in offspring
Note: Factor 1 was identical for both Profiles

Factor 2: Detection and efficiency of procedure

1. Detect new/rare mutations in patients

who present an uncommon or unclear phenotype

2. Allow storage of data, and hence test new mutations as they are discovered

3. Reduce the need for other medical investigations/procedures
Factor 2: Detection and efficiency of procedure

1. Identify a genetic risk for comorbidities

2. Reduce the need for other medical investigations/ procedures
aA two-factors solution was resolved in the analysis explaining 49.50 of the total variance. Analysis of the eigenvalues indicates that improvement of patients' and their proxies' well-being as well as detection and efficiency of WGS were perceived as most beneficial. These factors accounted for $25.30 \%$ and $24.20 \%$ respectively.

bA two-factors solution was resolved in the analysis explaining 79.61 of the total variance. Analysis of the eigenvalues indicates that improvement of patients' proxies as well as detection, were perceived as most beneficial of WGS. These factors accounted for $46.30 \%$ and $24.95 \%$ respectively.

conditions. In comparison, neurologists who never used WGS tended not to know what to foresee and those with over 20 years experience tended to foresee more risks than benefits. This brings to the forefront the critical roles training and experience play in the application of NGS technologies in the practice of neurology influencing physicians' opinions and practices.

While the benefits are all directed towards the patients and difficult cases of diagnostics - as shown for instance by the positive impact WGS is expected to have on selecting epileptic patients as candidates for surgery - the risks most identified by respondents were of financial and legal nature for all respondents equally. This is also what comes out of Helman et $a^{27}$ in which neurologists explained that they often did not engage in the use of WGS because of its cost or they did so only when linked to research projects, because of difficulties in obtaining health insurance coverage. Considerations about social and psychological risks, such as stigma and discrimination or anxiety raised by test results prevailed over WGS' technological features and over recommendations of professional associations. In other words, the social and psychological impact of genetic results on respondents' patients that is, psychological well being, access to healthcare and social services, reducing the need for other medical investigations/ procedures, possible discrimination or stigmatization of patients or their family members, the potential for anxiety or guilt, lack of access to, or availability of, the test, the cost of WGS, the lack of specific insurance coverage and of insufficient reimbursement seem to be more important than the technological advances WGS has to offer and also more important than what would be established by professional guidelines when respondents have to decide to request a WGS test for their patients. Our results show that while expressing a need for guidelines from professional associations, respondents would nonetheless be inclined to have flexibility in the application of professional recommendations for the use of WGS and WES in their practice. This is especially relevant because there is a precedent of establishing procedures on the management of WES and WGS results that were 
the subject of significant discussions in the medical community at the time such measures were made public. ${ }^{28}$ Notwithstanding their perception of risks and benefits, respondents foresaw an increased demand for targeted sequencing. They likewise expected WES and WGS to gain ground over other genetic tests. In fact, they reported it was not uncommon for patients to ask to undergo genetic tests. With increased access to direct-to-consumer genetic testing, even across jurisdictions where relevant legislation was either absent or varied widely, respondents expected more patients would consult their physicians about results obtained elsewhere. ${ }^{29,30}$ In our study, while we recruited neurologists located in low-, middle- and high-income countries, where access to new technologies can differ significantly,

Table 3 Concerns about the use of WGS in clinical practice by Profile

\begin{tabular}{|c|c|}
\hline Profile $A^{\mathrm{a}}$ & Profile $B^{\mathrm{b}}$ \\
\hline Factor 1: Patients and proxies & $\begin{array}{l}\text { Factor 1: Potential adverse effects for patients and proxies, and medical } \\
\text { professionals }\end{array}$ \\
\hline 1. Inform patient's relatives about their own genetic risks & 1. Patients'/proxies' difficulties in understanding the results \\
\hline 2. Patients'/proxies' difficulties in understanding the results & 2. Issues raised by the management of unexpected findings \\
\hline $\begin{array}{l}\text { 3. Need for further genetic testing of family members for appropriate interpretation } \\
\text { of results }\end{array}$ & $\begin{array}{l}\text { 3. Risk of discrimination or stigmatization of patients or their family members } \\
\text { 4. Inform patient's relatives about their genetic risks }\end{array}$ \\
\hline $\begin{array}{l}\text { 4. Need to re-contact patients to inform them about newly discovered mutations } \\
\text { linked to their neurological condition }\end{array}$ & $\begin{array}{l}\text { 5. Need to re-contact patients to inform them about newly discovered mutations } \\
\text { linked to their neurological condition }\end{array}$ \\
\hline Factor 2: Lack of resources & Factor 2: Lack of resources \\
\hline 1. Lack of access to/availability of the test & 1. Potential for causing emotional harm to patients or proxies \\
\hline 2. Cost/lack of insurance coverage and reimbursement & 2. Lack of time and resources to educate/inform patients/proxies about the test \\
\hline 3. Lack of specific clinical guidelines to use WGS in practice & and its results \\
\hline \multirow{5}{*}{$\begin{array}{l}\text { 4. Obligations to follow overly constrictive professional guidelines that could limit } \\
\text { choices in practice } \\
\text { 5. Lack of time and resources (eg, genetic counselling) to educate/inform patients/ } \\
\text { proxies about the test and its results }\end{array}$} & 3. Lack of specific clinical guidelines to use WGS in practice \\
\hline & 4. Obligations to follow overly constrictive professional guidelines that could limit \\
\hline & choices in the practice \\
\hline & 5. Lack of access to/availability of the test \\
\hline & 6. Costs/lack of insurance coverage and reimbursement \\
\hline
\end{tabular}

Factor 3: Potential adverse effects for patients and medical professionals

1. Professional liability for adverse outcomes that might occur as a result of

returning (or not) the test findings

2. Risk of discrimination or stigmatization of patients or their family members

3. Potential for causing emotional harm to patients or proxies (anxiety, guilt, etc.)

aConcerns about WGS were characterized by a three factors explaining $74.90 \%$ of the total variance: concerns for patients' proxies ( $28.11 \%)$, lack of resources (26.80\%) and potential for adverse effects $(19.99 \%)$

${ }^{b}$ Concerns about WGS were characterized by two factors - explaining $83.32 \%$ of the total variance - including potential adverse effects (53.64\%), and lack of resources (29.68\%).

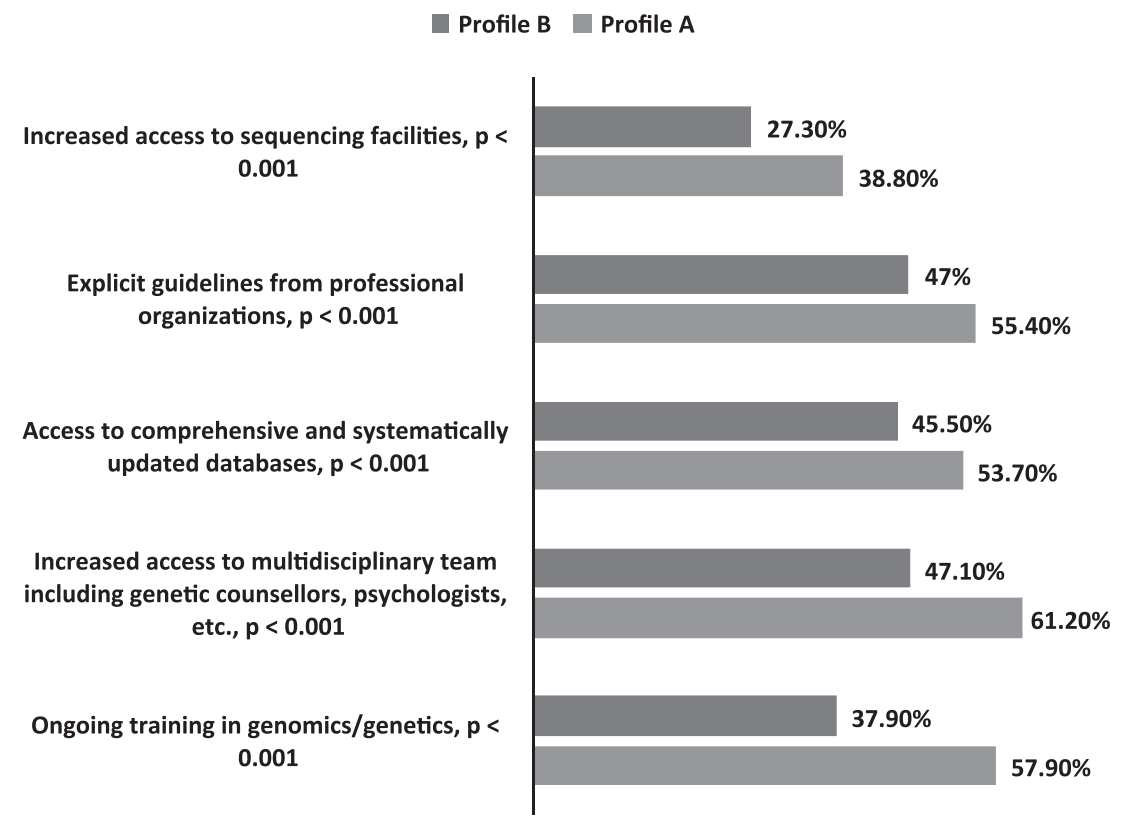

Figure 3 Need of resources to use WGS in medical practice per profile. 
because of an under-representation of many countries and geographical areas, we cannot determine if the availability of new diagnostic and treatment tools may have influenced participants' responses with respect to the foreseeable use of NGS technologies.

Factors influencing the use of genetic testing may not be restricted to the training of physicians nor the availability of tests. Yoshida et al ${ }^{31}$ showed that neurologists in Japan are likely to conduct genetic testing based on the characteristics of the diseases, and the circumstances surrounding their patients and families. Our results are concordant with those of Salm et al and Dominguez-Carral et al. ${ }^{32,33}$ In Salm's study, US neurologists and psychiatrists reported deficits in their knowledge of genetics as applied to their medical practice, and a lack of access to genetic counselors or geneticists to whom they could refer their patients. ${ }^{32}$ Dominguez-Carral et $a l^{33}$ in a recent survey conducted among child neurologists in Spain, also highlight the need these professionals have for continuing education programs and support from geneticists.

\section{Resources that would help integrate WGS into clinical practice}

The mapped two profiles of respondents appeared in low-, middle-, and high-income countries, which would indicate that the availability of this test (ie, cost and access) was not a determining factor in their perceptions. Profile A respondents had more training in genetics and felt more informed than their counterparts. More experience and knowledge may explain, for instance, why more of them acknowledged the benefits of WGS based on genetic data storage, without the need for further samples whenever new mutations were discovered.

Concerns on the use of WGS were different between the two Profiles, which may reflect their difference in experience and knowledge. Profile A, in comparison to Profile B, might know, based on their experience using genetic testing that there could be a need for further tests to improve the interpretation of results. Experience, or the lack thereof, may explain why Profile B respondents do not perceive the advantage that the use of WGS and other NGS technologies provide, by allowing the storage of raw sequencing data, thus avoiding test repetition. A second Profile A concern was the possible professional liability for adverse outcomes resulting from the return (or not) of test findings. In this regard, unambiguous rules on the management of findings, expected or otherwise, would clarify the responsibilities of physicians on the communication of any type of genetic result. Last, and independently of professional liability, Profile $\mathrm{B}$ was more concerned about the management of incidental findings than Profile A. On the basis of the intense debate at various professional levels on this subject, we expected this issue to be of interest for all neurologists participating in our survey. We understand that legal responsibilities are only one aspect of sharing genetic results with patients. The results of our survey supplement those of Townsend et $a l^{7}$, where health professionals expressed their preference for targeted analysis of WGS results, thus limiting the volume of data handling and so of incidental findings being generated, decreasing eventually the 'burden' of overseeing the return of said results.

Management of unexpected findings involves all the organizational aspects of conveying such information, and includes the availability of multidisciplinary teams and a health system capable of managing additional diagnostic procedures and treatments for unforeseen medical conditions. ${ }^{34}$ Our entire sample expressed concerns about the impact of WGS on patients and their immediate families regarding communication of results, understanding of information, recontacting patients, and the risks of discrimination and stigmatization. These findings matched results of another study, where neurologists expressed concerns about the potential for psychological harm when genetic information reached patients. ${ }^{32}$ Some neurological disorders, such as epilepsy, may carry additional burdens influenced by age of onset, severity and frequency of seizures, depression, anxiety, or cognitive deficits, and influence patients' family-planning process. $^{35-37}$

Neurologists participating in our study who were more supportive of the use of WGS were also those who expressed a significantly higher need for resources to help in this endeavour. This was also the case in Sharp et al, ${ }^{38}$ where 'physicians agreed that new findings in genetics were changing clinical practice in their areas of medicine, and that increasing their familiarity with genetics would benefit their patients'. We share Sharp et al's reflection on the need for 'think(ing) creatively' on how to incorporate genetics education into physician training.

The implementation of resources entails the intervention of various stakeholders on the use of WGS in the practice of neurology. While training in genomics/genetics is part of the curriculum of educational institutions, in our view medical associations should be the ones who guarantee accessible, ongoing training for medical professionals. Our respondents expressed a need for explicit guidelines from professional organizations in regulating the use of NGS technologies in their medical practice. Yet, these guidelines may need to be flexible and attuned to the reality of medical practice, local healthcare policies, access to medical care and treatment, and the social support needed by affected individuals and their families at time of confirmation of suspected and/or newly-discovered health conditions. Hospital and academic institution policies could also be developed in order to create opportunities for neurologists to work with multidisciplinary teams, including geneticists, genetic counsellors or psychologists, to facilitate the understanding of genetic results. Access to sequencing facilities and to comprehensive and systematically updated databases could also be beneficial to improve diagnoses and the treatment of patients. Covering the bridge between bench work and medical practice requires commitment at higher levels of healthcare management to support both basic research and medical training and a productive link between the two. Moreover, such support should be extended to obtain - and take into consideration - information about patient expectations and preferences on genetic testing.

\section{Limitations}

Our study aimed to include the opinions of neurologists worldwide. Although invitations were issued in English, French, and Spanish, we acknowledge that presenting our survey in English exclusively may have prevented broader participation. In addition to the language barrier, expertise, access to genetic testing, as well as cultural background may have ultimately influenced the decision to participate, or not, in our survey. Lastly, our results represent those neurologists who actually responded and thus are interested in the subject of this survey and wanted their voices to be heard. A higher rate of participation in general, and a more prominent representation of neurologists with specialties other than epilepsy, might have led to different results.

\section{CONCLUSION}

According to the perception of participating neurologists, there could be gaps in organization, funding and education in support of WGS in clinical settings. Considering that several neurological conditions have a genetic background, the use of WGS could strongly contribute to a faster and more precise diagnosis and possibly better treatment. Our results highlight the potential need for institutional policies in establishing collaborative working teams and for a stronger role for professional associations. The latter could be by providing clearer guidelines and educational tools to help physicians using WGS in their 
practice. Lastly, our results indicate the need for financial and organizational resources for the use of WGS in medical practice, and the subsequent management of newly discovered medical conditions. The challenges of using WGS in clinical practice are substantial. Our findings could be useful by being extrapolated to the practice of neurology in general as well as to other medical specialities where WGS may likewise play an increasingly useful role.

\section{CONFLICT OF INTEREST}

The authors declare no conflict of interest.

\section{ACKNOWLEDGEMENTS}

We thank Mr Jean Poupart for his contribution in the recruitment process, Mr John Bisping for helpful discussion, and the IT department at Université de Montréal, Public Health Research Institute (IRSPUM) for on-line questionnaires, data collection and statistical analysis. We are indebted to the medical associations worldwide that helped distributing our survey, as well as to the neurologists pre-testing our questionnaire, and all those responding to our survey. We gratefully acknowledge the support of the Canadian Institutes of Health Research (CIHR), Génome Québec and Genome Canada.

1 Jarvik GP, Amendola LM, Berg JS et al: Return of genomic results to research participants: the floor, the ceiling, and the choices in between. Am J Hum Genet 2014; 94: 818-826.

2 Korol S, Hurlimann T, Godard B, de Denus S: Disclosure of individual pharmacogenomic results in research projects: when and what kind of information to return to research participants. Pharmacogenomics 2013; 14: 675-688.

3 Mathieu G, Groisman IJ, Godard B: Next generation sequencing in psychiatric research: what study participants need to know about research findings. Int J Neuropsychopharmacol 2013; 16: 2119-2127.

4 Hallowell N, Hall A, Alberg C, Zimmern R: Revealing the results of whole-genome sequencing and whole-exome sequencing in research and clinical investigations: some ethical issues. J Med Ethics 2015; 41: 317-321.

5 Hegde M, Bale S, Bayrak-Toydemir P et al: Reporting incidental findings in genomic scale clinical sequencing-a clinical laboratory perspective: a report of the Association for Molecular Pathology. J Mol Diagnos 2015; 17: 107-117.

6 Lohn Z, Adam S, Birch P, Townsend A, Friedman J: Genetics professionals' perspectives on reporting incidental findings from clinical genome-wide sequencing. Am J Med Genet A 2013; 161A: 542-549.

7 Townsend A, Adam S, Birch PH, Lohn Z, Rousseau F, Friedman JM: 'I want to know what's in Pandora's Box': comparing stakeholder perspectives on incidental findings in clinical whole genomic sequencing. Am J Med Genet A 2012; 158A: 2519-2525.

8 Zawati MH, Parry D, Thorogood A et al: Reporting results from whole-genome and whole-exome sequencing in clinical practice: a proposal for Canada? J Med Genet 2014; 51: 68-70.

9 de Koning TJ, Tijssen MA: Movement disorders in 2014. Genetic advances spark a revolution in dystonia phenotyping. Nat Rev Neurol 2015; 11: 78-79.

10 Swerdlow NR, Gur RE, Braff DL: Consortium on the Genetics of Schizophrenia (COGS) assessment of endophenotypes for schizophrenia: an introduction to this. Schizophr Res 2015; 163: 9-16.

11 LePichon JB, Saunders CJ, Soden SE: The Future of Next-Generation Sequencing in Neurology. JAMA Neurol 2015; 72: 971-972.

12 Matthews PM, Edison P, Geraghty OC, Johnson MR: The emerging agenda of stratified medicine in neurology. Nat Rev Neurol 2014; 10: 15-26.

13 Gahl WA, Markello TC, Toro C et al: The National Institutes of Health Undiagnosed Diseases Program: insights into rare diseases. Genet Med: Off J Am Coll Med Genet 2012; 14: 51-59.
14 Foo JN, Liu J, Tan EK: Next-generation sequencing diagnostics for neurological diseases/disorders: from a clinical perspective. Hum Genet 2013; 132: 721-734.

15 Halvardson J, Zhao JJ, Zaghlool A et al: Mutations in HECW2 are associated with intellectual disability and epilepsy. J Med Genet 2016; 53: 697-704.

16 Chen Z, Wang JL, Tang BS et al: Using next-generation sequencing as a genetic diagnostic tool in rare autosomal recessive neurologic Mendelian disorders. Neurobiol Aging 2013; 34: e2411-e2447.

17 Jiang T, Tan MS, Tan L, Yu JT: Application of next-generation sequencing technologies in Neurology. Mol Neurol 2013; 2: 125.

18 Nemeth AH, Kwasniewska AC, Lise S et al: Next generation sequencing for molecular diagnosis of neurological disorders using ataxias as a model. Brain 2013; 136: 3106-3118.

19 Veeramah KR, Johnstone L, Karafet TM et al: Exome sequencing reveals new causal mutations in children with epileptic encephalopathies. Epilepsia 2013; 54: $1270-1281$.

20 Hurlimann T, Jaitovich Groisman I, Godard B: The elusive ideal of inclusiveness: lessons from a worldwide survey of neurologists on the ethical issues raised by wholegenome sequencing. BMC Medical Ethics 2017; 18: 28.

21 Foo JN, Liu JJ, Tan EK: Whole-genome and whole-exome sequencing in neurological diseases. Nat Rev Neurol 2012; 8: 508-517.

22 Keogh MJ, Chinnery PF: Next generation sequencing for neurological diseases: new hope or new hype? Clin Neurol Neurosurg 2013; 115: 948-953.

23 Sherr EH, Michelson DJ, Shevell MI, Moeschler JB, Gropman AL, Ashwal S: Neurodevelopmental disorders and genetic testing: Current approaches and future advances. Ann Neurol 2013; 74: 164-170.

24 Foley SB, Rios JJ, Mgbemena VE et al: Use of Whole Genome Sequencing for Diagnosis and Discovery in the Cancer Genetics Clinic. EBioMedicine 2015; 2: 74-81.

25 Groisman IJ, Mathieu G, Godard B: Use of next generation sequencing technologies in research and beyond: are participants with mental health disorders fully protected? BMC Med Ethics 2012; 13: 36.

26 Lantos JD, Artman M, Kingsmore SF: Ethical considerations associated with clinical use of next-generation sequencing in children. J Pediatr 2011; 159: e871.

27 Helman G, Bonkowsky JL, Vanderver A: Neurologist comfort in the use of nextgeneration sequencing diagnostics: current state and future prospects. JAMA Neurol 2016; 73: 621-622.

28 Presidential Commission for the Study of Bioethical Issues. Anticipate and Communicate: Ethical Management of Incidental and Secondary Findings in the Clinical, Research, and Direct-to-Consumer Contexts. Report of the Presidential Commission for the Study of Bioethical Issues. Presidential Commission for the Study of Bioethical Issues: Washington, DC, USA, 2013.

29 Borry $\mathrm{P}$, van Hellemondt RE, Sprumont $\mathrm{D}$ et al: Legislation on direct-to-consumer genetic testing in seven European countries. Eur J Hum Genet 2012; 20: 715-721.

30 Niemiec E, Howard HC: Ethical issues in consumer genome sequencing: Use of consumers' samples and data. Appl Trans/ Genomics 2016; 8: 23-30.

31 Yoshida K, Ohata T, Muto K et al: Survey on the attitude toward genetic testing of neurologists certified by the Japanese Society of Neurology. Clin Neurol 2013; 53 : 337-344.

32 Salm M, Abbate K, Appelbaum P et al: Use of genetic tests among neurologists and psychiatrists: knowledge, attitudes, behaviors, and needs for training. J Genet Counsel 2014; 23: 156-163.

33 Dominguez-Carral J, Lopez-Pison J, Macaya A, Bueno Campana M, Garcia-Perez MA, Natera-de Benito D: Genetic testing among Spanish pediatric neurologists: Knowledge, attitudes and practices. Eur J Med Genet 2017; 60: 124-129.

34 Dal-Re R, Katsanis N, Katsanis S, Parker LS, Ayuso C: Managing incidental genomic findings in clinical trials: fulfillment of the principle of justice. PLoS Med 2014; 11: e1001584

35 Camfield PR, Camfield CS: What happens to children with epilepsy when they become adults? Some facts and opinions. Pediatr Neurol 2014; 51: 17-23.

36 Helbig KL, Bernhardt BA, Conway LJ, Valverde KD, Helbig I, Sperling MR: Genetic risk perception and reproductive decision making among people with epilepsy. Epilepsia 2010; 51: 1874-1877.

37 Maroufi A, Khomand P, Ahmadiani S, Alizadeh NS, Gharibi F: Prevalence and quality of anxiety in patients with epilepsy. Epilepsy Behav 2014; 32: 34-37.

38 Sharp RR, Goldlust ME, Eng C: Addressing gaps in physician education using personal genomic testing. Genet Med: Off J Am Coll Med Genet 2011; 13: 750-751.

Supplementary Information accompanies this paper on European Journal of Human Genetics website (http://www.nature.com/ejhg) 